\title{
Family caregivers-of patients with chronic renal failure terminal psychological stress
}

\begin{abstract}
Caregivers of Patients with Chronic Renal Failure Terminall suffer impairment in psychological and social order due to the unlikelihood of recovery as well as the uncertain and progressive course of this disease. In the municipality Guisa, Granma Province, there is a health problem regarding this issue, caregivers-relatives of patients with chronic renal failure Terminal (grades 4 and 5 of the disease in hemodialysis treatment) of the municipality constantly attend consultations Psychology and Psychiatry, specialist for help refer feel as stressed. Why this research shows held under the title "family caregivers of patients with end-chronic renal failure, the municipality Guise psychological stress" in order to determine the main manifestations of psychological stress in these caregivers. analysis-synthesis and inductive-deductive and the following techniques psychological evaluation: document review, interviews, observation, Anxiety Scale (STAI), Beck Inventory, Scale to comply with the same theoretical methods were applied Zarit and symptomatic Stress Scale. The results stated that the main manifestations of psychological stress of these caregivers are anxiety, depression, and loss of appetite, sleep disturbances, heartburn, fatigue, sweating and irritability. This research work has vital importance
\end{abstract}

Keywords: psychological stress, family caregivers, kidney failure
Volume 5 Issue 5 - 2018

\author{
Yanelys Taset Alvarez,' Lic Lianna Lady \\ Martinez Fajardo ${ }^{2}$ \\ 'University of Granma, Cuba \\ ${ }^{2}$ Mental Health Center, Cuba
}

Correspondence: Yanelys Taset Alvarez, University of Granma, Manzanillo Highway, Km 171\%2, Peralejo, Granma, Bayamo, Cuba, Email:ytaseta@udg.co.cu,nefty@nauta.cu

Received: May 17, 2018 | Published: September 17, 2018

\section{Introduction}

The human being is a unit where biopsicosocial shows the dialectic interrelationship between soma and psyche through a systemic functioning. Among the psychosocial factors you can find a range of phenomena involved in the health - disease process, because this is not a phenomenon that occurs in the abstract but in a particular individual. Customs, beliefs, social representations, attitudes, systems of relationships and social support, as well as the ways and lifestyles influence the thinking, feeling and acting of the subjects, speaking in the context of their physical, social environment and cultural. This affects not only the world view of individuals, but in the perception and experiences that can have at any given time relationship health disease.

In the Chronic Noncommunicable Diseases (NCDs), the relationship between the biological and social evidenced, for as scourge of the new century, are closely linked to the lifestyle that people assume. The condition of these diseases is mostly curable. It exists in the world an estimated $60 \%$ of people are carriers of chronic diseases and increased incidence Cuba increasingly. Within the CNCD are the IRC, conceived as an irreversible disease, and diffuse bilateral renal function, which usually evolves slowly and progressively, until the state of terminal uremia, with all its complications damage. Its main risk factors are: Hypertension, Diabetes Mellitus, hypercholesterolemia, among others.

CKD patients undergoing treatment with alternative methods of renal function (hemodialysis, peritoneal dialysis and renal transplant function) are approximately two million worldwide. In developed countries the prevalence rate is approximately 500-1400 patients per million populations. The incidence is approximately $300-340$ patients per million population and increases around $10 \%$ annually.
In the Latin America prevalence is 447 patients per million populations. In Cuba, the prevalence is 225 patients per million population and patients with CKD at different stages of development of the disease for a total of 40,000 patients; It is considered the $7^{\text {th }}$ leading cause of death but is linked to the main causes of death, because death is involved primarily in patients suffering from Diabetes Mellitus, Hypertension Disease and Malignancies.

In Granma province, the Department of Nephrology of Carlos Manuel Hospital de Céspedes currently serves 113 patients with Chronic Renal Failure (ESRF) with criteria hemodialysis treatment, i.e. those patients who are in groups 4 and 5 of this disease.

In the municipality of Guise in that province there is an alarming situation, $35 \%$ of the population is at risk population (Annex \#1) and of this $31 \%$ is hypertensive, non-communicable chronic disease that behaves as one of the main risk factors for CKD. In this regard, it is one of the conditions prevailing in the municipality, there are currently 20 nephrotic and these 9 with IRCT, catastrophic illness increased risk of medical complications, ${ }^{2}$ hospitalizations, mortality, economic impact on an individual and social level, the suffering and progressive deterioration resulting in patients and family impact.

The higher cost of this disease is, undoubtedly, its human cost, the dimensions are invaluable, and within them in a special way: caregivers. The caregiver often has to interrupt his usual pace and limit their social life, which brings eventually a negative impact on their health from all orders, as devoted years of his life to give affection and care for her sick, that with the advance of the disease, will be unable to reciprocate this commitment and that often react aggressively and inappropriate behaviour.

Care for patients with chronic renal failure Terminal represents a psychological ${ }^{3}$ burden for the caregiver and the family, based 
on the different problems they have to face such as impact on the patient and the family generated by the hemodialysis treatment for 4-5 hours 3 times a week and lifetime, real risk of infection and other serious complications in patient preparation for submission to it (catheterization and arteriovenous fistulas) long-hospital stays, financial burden, isolation and high levels of psychological stress especially if the caregiver is a relative of the patient.

In most cases, care is borne by mothers, wives, daughters, daughters or sisters. In the country, studies show that the lowest of women who are responsible to care for the sick percentage was about $70 \%$.

After reviewing documents such as medical records of patients with debugger treatment and leaves by consultations of Psychology and Psychiatry at the municipality Guise, it was obtained that $100 \%$ of caregivers of patients with ESRD are female relatives (daughters, mothers, sisters and wives) in middle adulthood as developmental stage. Despite the satisfaction felt by caregivers for care provided by the Ministry of Public Health, ${ }^{4}$ transport which guarantees the transfer of the patient to the systematic use of hemodialysis for free and priorities available to the service, constantly flock to consultation of Psychology and Psychiatry for help because lack of social recognition is found, they expressed feeling burdened, isolated, lack of social, tense and stressed support. ${ }^{5}$ Due to the care of the patient and family environment to which they are exposed often debuting major diseases among which are chronic non-communicable as Hypertension, Diabetes Mellitus, gastroduodenitis, peptic ulcer Gastroduodenal among others that are now a health issue in the municipality, in this regard, considering the above, the caregiver who cares?

Known authors of national and international literature has been devoted to the study of the impact of the quality of life of caregivers, where they have arrived at conclusions as:

A. Espin (2001) raises the loss of relationship with the patient progressively isolated and living in another world, which is not known as access leads to mental solitude of the caretaker, who is total, when only the sick in physical presence and is only able to capture the affection and not to give his.

Aymara Reyes Saborit (2006) notes that the ethical implications of care in patients with NCDs are indubitable, a situation that causes a state of tension and anxiety in the caregiver in which prevalent symptoms such as depression, anxiety and emotional lability.

L. Araya, M. Guaman, M. \& Godo, C. (2007) are of the opinion that the family and especially the caretaker-family suffer an impact on the economic order to be limited resources, because on the one hand the caregiver often forced to stop working away from home to pursue the sick, on the other hand, food and medication costs increase.

Reyes, 2007; Arraras and Garrido (2008) suggest that primary caregivers exposed to anxiety and constant stress are more likely to exhibit sleep disturbances, malnutrition or malnutrition, ${ }^{6}$ physical inactivity and consumption of alcohol, nicotine, which affects your immune function and has impact on their health.

Dr. Matías Guiu, Chief of Neurology, (2009) reports that people who devote themselves to the care of their families usually suffer from depressive disorders ( 70 percent of cases), reflected in the moral pain, sleep disorders and digestive problems. It stresses that the stress of this dedication can be a precipitating factor of many diseases.
The use of the term stress has become popular without most people are clear what the same. In reviewing the extensive literature on the subject, there are many definitions some of which approach it either from the perspective of stress as a stimulus, response or result.

Selye (1966), considered the father of stress, introduced the concept as a set of non-specific physiological reactions of the organism to various harmful agents in the environment of physical or chemical nature, defining with it a coordinated responses set against any form of noxious stimulus (including psychological threats), a reaction he called the General Adaptation Syndrome.

Richard Lazarus (1983) presented an important contribution: "Stress is the result of the relationship between the individual and the environment, assessed by that as threatening, overflowing its resources and puts his welfare at risk." This author makes important contributions to defining stress, but its exhibits highlights the importance he attached to the outside, ie states that the environment is the main factor for the presence of stress occurs, minimizing the role play the personal characteristics of the subjects.

After reviewing the definitions of stress, in recent years it has proved how it has been changing the concept to position itself today as a process in which multiple factors of various kinds such as developmental stage, sex, level of education, socio-historical among others. conditions The author of this paper considers that the social historical context in which they live, taking as sustenance each of the valuable involved capital theories in the world of scientific research, stress should be analyzed with a focus or integrated look that allows distinguish the subject their person logical resources as generators stimuli responses continuous and active adaptation, because every day he will find himself immersed in the social environment and in turn, is the protagonist par excellence of it.

Thus, from an integrative perspective and consider appropriate for the purposes of this investigation was decided to work with the concept of stress offers Zaldivar (1996), which assumes that stress is an experiential state unpleasantness sustained over time, accompanied greater or lesser extent psychophysiological disorders arising in an individual as a consequence of alteration of its relationship with the environment, which requires the subject demands or requirements of objectively or subjectively which, are threatening to it and over which it has or believed to have little or no control."

Based on this conceptualization of psychological stress and the problem situation posed: What are the main manifestations of psychological stress on caregivers-relatives of patients with chronic renal failure Terminal, the municipality Guise? Constituting this research question of this study, which is proposed as an objective: to determine the main manifestations of psychological stress in these caregivers.

This research is desirable as it will determine the main manifestations of psychological stress of caregivers-relatives of patients with ESRD, the municipality Guise in order to lay foundations to the development of a strategy of psychological intervention in order to reduce high levels stress they are subjected these people. It is socially relevant and to the extent that these manifestations of stress are known, as well as the psychological strategies, actions that facilitate the intervention and / or control of chronic non-communicable (consequences of stress) as hypertension will be implemented, Diabetes mellitus, Peptic Ulcers, among others. 
Considering further that until now, have not conducted research related to the existence of manifestations of psychological stress on caregivers-relatives of patients with ESRD, the research will obtain new results that will impact positively on both caregivers and chronic renal patients.

\section{Method}

Participants: Inclusion criteria were the universe

a. Being familiar caregiver of a patient with Chronic Renal Failure Terminal.

b. Willingness to participate in the investigation.

The research universe was made up of 9 family caregivers of a patient with Chronic Renal Failure Terminal municipality of Guise.

Variable under study: conceptual and operational definition

Psychological stress: "It is an unpleasant experiential state is sustained over time, accompanied in varying degrees of psycho physiological disorders arising in an individual as a result of altering their relationships with the environment imposed by the subject demands or requirements, objectively or subjectively, are threatening him and on which you have or think they have little or no control "(Zaldivar, 1996).

This variable assumes different dimensions expression

\section{Dimensions}

Physiological: Set of effects that occur in the body affect him health and causing discomfort for the presence of symptoms the person.

\section{Indicators}

a) Disorders or heart ailments product Situational discomforts personally experienced: cardiovascular disorders.

b) Gastrointestinal disorders: disorders or conditions that have to do with the digestive system caused by imbalances in digestion of food as a result of emotional discomfort.

c) Skin disorders: disorders or skin conditions.

Psychical: Set of effects that occur in the subjective world of the subject as a result of the action of harmful environmental elements that affect their psychosocial development.

\section{Indicators}

a) Anxiety is a reaction of the organism when it has lost its chance organized and coordinated response. It has psychological somatic manifestations, like shortness of breath, discomfort, uncertainty, inability to perform a task.

b) Depression: Feelings of sadness unmotivated upsets and suffering, accompanied by anxiety, feelings of worthlessness, guilt. The depressed person subavalora, ideas manifest selfaccusation.

c) Fatigue: state of exhaustion generated tensions maintained by long periods of time.

d) Extreme fatigue: Decreased excessive forces to carry out a task.

Behaviour: Set of events that affect the behaviour of the subject.

\section{Indicators}

a) Irritability: Lowering the threshold for impressionability and emotional responsibility. Little emotional control.

b) Muscle tension Muscle contractions in the body accompanied by pain that prevents the performance of the activity.

\section{Methods and techniques}

Analysis-Synthesis and Induction-Deduction: They were used throughout the research process, with vital importance in building the conceptual framework and the analysis of results.

\section{Techniques}

\section{Observation}

Observation is a cognitive, interested and attentive data search offered. The same was done with a guide to flexible working; I aimed to observe in depth the possible manifestations to speak in favour of the existence of stress in the behaviour of caregivers-relatives of patients with ESRD, based on the assessment of skills personal that they have them. Observation will be included and covert.

\section{Interview}

The interview was used to support and complement the results of the investigation. He allowed greater trust and empathy with the interviewee contributing to this is expressed more freely, while allowing that through the corporeal language (gestures, intonation, facial expression, etc.) can be detected information that is beyond speech.

\section{Initial interview}

It is the first contact with caregivers-relatives of patients with ESRD. Its main objective is the presentation of research and knows their attitude towards the process. It also seeks to obtain a general impression of the life of every caregiver, as it possible to measure socio-demographic variables (age, sex, marital status, education,) and other related kinship, number of family members living at home, data state of health, time spent by the patient and the experiences that expose the fact perform this task. It is possible to establish the rapport and empathy.

\section{Depth interview}

It was applied in order to know in depth the general characteristics of caregivers and determine which are the main events that speak in favour of the existence of psychological stress.

It was assessed on physiological reactions in caregivers-relatives, i.e. discomfort and suffering that the subject interviewed, as well as linking these discomforts and suffering with the existence of psychological stress. He also sought to determine individual potential it has for addressing this problem and has knowledge on the subject matter and personal health. ${ }^{7}$ Other features were also evaluated. Assesses the social integration of individuals with their environment, i.e. the quality of interpersonal relationships with those around you.

\section{Trait anxiety inventory and as a state (STAI)}

It allowed us to evaluate the levels of anxiety as a state and as a trait according to corroborate the anxious manifestations reported by caregivers-relatives of patients with ESRD, who can speak in 
favour of the existence of psychological stress. It should be noted that subjects who achieve high marks in trait anxiety are more likely to get a high evaluation anxiety as a state, in stressful situations.

This technique theoretically considered anxiety with two forms of expression, as a state and as a trait. It applies to adults and adolescents, healthy or psychiatric disorders, both individually and collectively. Each scale is presented separately to the subject form the first state and then the trait, each with 20 items that record various manifestations of anxiety, in a gradient of four points. On the scale of state he is oriented to the subject must respond as you feel at the moment in relation to the formulated items, and how it feels genealmente in relation to the items on the scale of trait anxiety. (See Annex \#6).

To qualify you must know the score achieved on each item and use a key to define which group entries are added. A formula is then used, whose final result allows to locate the subject in different levels of anxiety for each scale, which is defined as low, medium and high.

\section{Anxiety as a state}

Rating: Add the score marked by the subject.

Items $3,4,6,7,9,12,13,14,17,18=\mathrm{X}$

Items $1,2,5,8,10,11,15,16,19,20=\mathrm{Y}$

$(\mathrm{X}-\mathrm{Y})+50=$ anxiety level

LOW $(<30)$

MEDIUM (30-44)

High $(>45)$

\section{Trait anxiety}

Rating: Add the score marked by the subject.

Items $22,23,24,25,28,29,31,34,35,37,38,40=\mathrm{X}$

Items $21,26,27,30,33,36,39=\mathrm{Y}$

$(\mathrm{X}-\mathrm{Y})+35=$ anxiety level

LOW $(<30)$

MEDIUM (30-44)

$\operatorname{High}(>45)$

\section{Inventory Beck}

The Beck Depression Inventory was used to assess the depth of depression in caregivers-relatives of patients with ESRD. This scale was developed initially as an instrument of 21 categories of symptoms and attitudes, with 4 or 5 statements, and each reflects a growing degree of depth of depression. The content of the items reflect clearly the importance given by the authors to the cognitive component of depression, only a third of the items refer to physiological or behavioural aspects obtained scores range from 0 to 56. (See Annex \#7) 21 have dimensions that are:
a) Frame of mind
b) Pessimism
c) Failure
d) Discontent
e) Feelings of guilt
f) Need for punishment
g) Self hate
h) self-accusation
i) suicidal
j) Crying
k) Irritability
1) Social isolation
m) Inability decision
n) Body image
o) Inability to work
p) Sleep disorders
q) Fatigue
r) Loss of appetite
s) Weight loss
t) Hypochondria
u) Loss of libido

\section{Scale overload of Zarit Caregiver}

To determine the degree of load experienced by caregiversfamily Scale Caregiver Overload Zarit was used. This scale reflects a subjective approach to the load and, although this should be taken into account when interpreted, the results of different studies show that subjective burden is a good indicator of the effects of care. The original questionnaire contained 29 items designed to assess the subjective burden of care, taking into account the following aspects: physical and psychological health, economic and labor issues, social relations and relationship with the person receiving care increased. Responses to the items were added to obtain a single load index. Scores correlated significantly with load psychopathological symptoms of caregiver, with the mood and physical health of the caregiver, and the quality of the relationship between the caregiver and the care recipient. It is valid to clarify that this scale will be graded only qualitatively, as it does not constitute objective of this research to know the level of subjective burden of caregivers - family but rather, as the psychic burden to which they are subjected constantly determines the presence of manifestations of psychological stress.

\section{Symptomatic Stress Scale (SSS Questionnaire Seppo Aro)}

This instrument allowed the presence of subjective symptoms that develop on the basis of psychological stress on every caregiver. It also allows knowing by the incidence rate of the symptoms that occur, if the person is stressed or not. (See Appendix \#9).

It consists of 18 items which present symptoms mentioned in the stress response has four options each with a score when marked by the subject

Rarely or never $=0$

Sometimes $=1$

Often $=2$

Very often $=3$

When performing the summation result will be sought in the following range:

Less than 8: Normal 
9 to 10: Edge line

11 onwards: Subject stressed

\section{Procedures}

Four working sessions were designed, which were planned individually, taking into account the characteristics of caregivers and techniques.

\section{Session I}

Technique: Initial interview

This session was aimed at presenting to family caregivers in order to research and asked their willingness to participate in the same defined the universe of study. Empathic relationships and rapport necessary for the "commitment" to the process by family caregivers are established. The initial interview it possible to measure sociodemographic variables (age, sex, marital status, education) and other related kinship, number of family members living at home, the time devoted to patient data.

\section{Session \#2}

Technique: In-depth interview

In-depth interview had as main objective to explore what are the main events that speak in favour of the existence of psychological stress, and sought to determine the individual advantages that have to face this problem and the state of their relationships.

\section{Session \#3}

\section{Technique: IDARE}

IDARE pursued the implementation of anxiety explore family caregivers expression in two forms: as a state: assessing whether a transient emotional state and trait evaluating whether it is a feature person logical family caregiver.

\section{Technique: Beck Inventory}

The use of this technique allowed us to evaluate the depth or intensity of depression in family caregivers quickly and objectively through quantitative and qualitative rating of the instrument way.

\section{Session \#4}

\section{Technique: Zarit Scale}

It was used to explore the burden experienced by family caregivers from their subjective prism considering aspects: physical and psychological health, economic and labour issues, social relations and relationship with the older person receiving care.

\section{Technique: Symptomatic Stress Scale}

The application of this scale allowed the presence of subjective symptoms that develop on the basis of psychological stress on every family caregiver. Also seeks to investigate by the incidence rate of the symptoms presented, if the person is stressed or not.

Observation technique was used in all the sessions, in order to corroborate the information obtained between verbal language and extra verbal caregivers. Porte and appearance, personal hygiene, emotional expressions and concentration while performing techniques.

\section{Results}

During the exchange and discussion of the objectives a climate of rapport was achieved, subjects reported feeling motivated by research that was carried out and expressed their willingness to collaborate in everything necessary, as this benefited them to them as family caregivers. Which provided an opportunity to obtain excellent results through the methods and techniques applied? The analysis was performed by working sessions:

\section{Session I}

Initial interview:

With the application of these technique general data-family caregivers of patients with Chronic Renal Failure such as obtained:

They belong to middle adulthood as developmental stage, aged 41 and 58 years old. Three have a university level education accounting for $33 \%, 4$ carers $12^{\text {th }}$. Grade $44 \%$ and 2 caregivers 9 no. degree representing $22 \%$. It was found that a caregiver's mother, 2 daughters are patients, 2 sisters and 4 their wives.

Households are integrated between 2 and 5 members. Of these carers 3 have dedicated 5 years or more in the care of their family 3 take about 3 years, one of them for the second time with a brother and the same pathology (IRC) said: "I was about to go crazy" considering the performance of this role from previous stages about 6 years, 7 years and a caregiver more, 2 caregivers about 9 years. Occupationally currently they are delinked due to the care of their family except 2 (university) who have maintained the employment relationship.

Expressed satisfaction of care provided by the Ministry of Public Health as transport to ensure the patient the hemodialysis service in organized, systematic, drugs, free skilled care and receive haemodialysis treatment with a very expensive technology, among other needs. They report that they have made some essential goods for the care of these patients as wheelchairs and wedges.

9 caregivers suffering from a disease, mostly non-transmissible chronic diseases have made their debut during the period leading performing this task, 4 are hypertensive for $44 \%, 3$ diabetic representing 33\% (these two are also hypertensive) chronic gastritis and 2 present for $22 \%$ (one suffers hypertension) these two caregivers attend sick family 9 years.

Refer suffer from Hypertension and Diabetes Mellitus, Gastritis, frequently decompensate and relate to the care of sick family member. In this respect it is valid to note that the Hypertension and diabetes are risk factors of $\mathrm{CKD}$, which together with the age of these caregivers and the work carried out are considered eminent persons risk. Table \#1 (Appendix \#10) data to characterize each caregiver that the same is. $100 \%$ of carers known to be the IRC although the quality and accuracy of their answers vary the level of schooling, (3 university carers) correctly expressed the concept of IRC, referring to all aspects of scientific way, (4 women of grade 12) claimed an empirical concept of disease even mentioned essential elements thereof, and (2 caregivers with school level 9th grade) raised insufficient concepts although essentially dominate roughly the main characteristics of this disease. They perceive stress as a state of "anxiety", "despair" of "very tired", "overwork", "sadness".9 caregivers have experienced ever representing $100 \%$. 


\section{Session \# 2}

Depth interview:

In depth interview applied at 9-family caregivers of patients with ESRD was obtained that:

$100 \%$ of caregivers assess their state of regular health associated this to daily some discomfort, as they feel: high or low blood pressure, high blood sugar or low, headache, abdominal pain (intake), sacrolumbalgias, muscle aches and decay.

9 caregivers express feeling tense or altered for $100 \%$ of these caregivers $78 \%$ i.e. 7 devote all their time to caring for the family, they do not have enough support from other family members, one of the caretakers who it stays with employment relationship refers: "I have fear, I try to make the time and I ran into the house" is perceived, that although it is their duty to care for the family, this has absorbed him his personal life, because everything revolves around your performance as a caregiver, and this has brought fears, family conflicts affecting to some extent her role as mother and wife (this is also evident in the caregivers who have spent more time caring for their family). One of the wife of a patient, also takes care of her two brothers with nervous disorder refers: also show that this situation has caused the onset of disease: 4 with Hypertension (44\%), 3 Diabetes Mellitus (33\%) and 2 chronic gastritis $(22 \%)$, which were initially addressed but have neglected lack of time, because "there is always something to do" a phrase that was heard virtually all caregivers was: "... this disease is terrible ...'”

9 caregivers representing $100 \%$ state that when the patient drinks water, soda, milk, juices secretly, (should only consume 30 millilitres three times per day), vomit, have loss or excessive appetite, drink alcohol and/or smoke, often come to insult them, and express: "I do not do you case" "I turn my back and I go," perceiving that often cannot cope with setbacks generated by the disease, modification of lifestyle, behavioural disorders and daily chores that come up. Despite the day care, barely can sleep at night schedule, constituting this other cause of discomfort, specifically in caretakers who take 3 years caring for her family and those with employment relationship affecting the implementation of its activities.

In items 23 and 24 are seen as economic costs are an important element that can contribute to psychological stress on caregivers, since $100 \%$ reflect that is one of the things that causes discomfort for concern that this type of person required extreme care especially food bases of meats, vegetables and carbohydrates (rice, flour, meats), personal hygiene and clothing, because they are conducted with extreme frequency which brings the excessive consumption of soap, detergent and others, whose economy most often is not enough to meet these needs and when they are facing desperate situations that may contribute to the presence of stress it makes them depressed, anxious, and sometimes very irritable.

In $100 \%$ of respondents it was obtained that feel committed to their family, because they think it is their duty also claim that suffer a lot to see how your loved one is "falling apart", "destroying", "falling apart", " falling apart ".

$33 \%$ of caregivers-relatives know some techniques that help you at certain times to the handling of their family (Relaxation and psychotropic drugs) due to the ongoing consultations for help with psychologists and psychiatrists, as referred feeling: tense, tired, disturbed, sad, burdened and anxious.

also was observed behavioural characteristics of different patterns of behaviour, making evident the relationship between the behaviour pattern and stress essentially could perceive in caregivers with typical behaviours behaviour pattern A (low frustration tolerance, accelerated, need for social recognition, compulsive personality traits, persistent: in 6 caregivers) and patterns of type $\mathrm{C}$ behaviour (anxiety, depression, resignation introversion: 3 caretakers) as caretakers presenting these types of behavioural patterns ( $\mathrm{a}$ and $\mathrm{c}$ ) have a greater predisposition to stress.

In general $100 \%$ consider their performance as good caretaker but express that sometimes could be better, and 33\% valued their actions as very good, perceiving that he gave his best express "no one would like care I ". (These correspond to the caregivers of patients with more than 9 years of illness and mother of a sick).

\section{Session \#3}

\section{Trait Anxiety Inventory as and as a state (STAI):}

This technique was applied to them at 9 caregivers-relatives of patients with ESRD, was obtained as shown in table \#2 (see Appendix \#11) 3 caregivers expressed medium levels of trait anxiety and as a state, for $33 \%$ it is necessary to note that play this role three years ago. Six expressed high levels of trait anxiety and state, accounting for $67 \%$. Thus it was confirmed that $100 \%$ of caregivers-relatives are anxious, constituting this one of the manifestations that always accompany psychological stress. The average levels of anxiety as a state is confirmed, because of the family situation being experienced therefore are more vulnerable to experiencing various situational reactions to certain stimuli are altered destabilizing and provoke inappropriate behaviour;

In analyzing the results of the 6 caregivers about high levels of trait anxiety and state, scale state said, highlighting the direct items: $(6,7$, $9,14,17,18$ ) with maximum value revealing feel: agitated, disturbed, anxious, "to explode" and stunned, in inverse items are the following minimum values $(1,8,10,15,19)$, they reveal irritability, fear, fatigue, tension, insecurity and sadness.

The values achieved are astonishing in scale score trait. The direct items are: $(22,23,25,28,3234,35,40)$ which express: they get tired quickly, desire to mourn almost always, unhappiness, difficulty in making decisions, repressed contents, dissatisfaction and melancholy. 6 caregivers, who expressed high levels of anxiety, care for her sick for more than 5 to 9 years and older.

Obviously rigged the stage of the disease to their characteristics and the time you have to play the role of caregiver affects directly with expressed anxiety levels and higher scores.

9 carers together representing $100 \%$ mentally anticipate about what can happen to your family at later stages, appreciating fears and future uncertainty.

\section{Session \#4}

\section{Beck inventory:}

The application of this technique showed that three caregiversrelatives of patients with ESRD presented a score between 21 and 25 points, indicating that $33 \%$ of caregivers have a moderate level of depression, while the remaining caregivers the $67 \%$, values 
between 30 and 48 points were considered throwing severe levels of depression. This is an alarming situation for the rapid progress that has this disorder and its consequences for both the caregiver and the patient. Behaviour size of the depression obtained in this technique is shown.

With this inventory data from other techniques that speak in favour of the presence of subjective symptoms of psychological stress they were corroborated.

as it was noted that other techniques the influence of age, education level and stage of disease in subjective well-being of caregiversrelatives as caretakers highest scoring match which expressed high levels of anxiety, in this case, caring for the sick for 5 years or more, as they have dedicated their life time appreciating this work exhaustion and stress maintained for long periods of time, muscle tension and loss of appetite that has generated weight loss. One of the caregivers expressed suicidal impulses, it should be noted that plays this role again with another brother and the same disease (ESRD) this is the only one that exists.

These results show that expressed by Ovidia Rodriguez Mendez (2005) who states that the presence of painful events as the death of a close relative, is a predisponible factor of suicidal behaviour. Similarly is I corroborates the statement made by Dr. Matías Guiu, Chief of Neurology, (2009) when referring to people who are dedicated to the care of their families usually suffer depressive symptoms (70\% of cases), reflected in sleep disorders and digestive problems. Statistical analysis of this technique was performed using fashion as a measure of central tendency, with which it could be inferred five modes: crying, mood disorders state of sleep, irritability and fatigability, i.e. is plurimodal.

\section{Session \#5}

Zarit scale on caregiver burden: With the application of this scale data obtained in the initial interview and depth as well as other techniques applied they were corroborated.

It was found that 9 caretakers representing $100 \%$, think that caring for his family he occupies most of his time, feeling overwhelmed by trying to combine it with other responsibilities, negatively affecting their relationships with other family members, which brings them feel tense or angry sometimes when they are near the sick. (Items 2, $3,5,6,9)$.

$78 \%$ of the population (7cuidadoras) consider their social life has been affected by the illness of his family, and their intimacy and friendship relations (items 11, 12, 13) .THE 100\% of caregivers think his health has worsened since caring for patients with IRCT therefore neglected flattering behaviours that affect health (item 10). 9 caretakers representing $100 \%$ believe that the feeling of sick family depends on them, a situation that compromises feel that sometimes would drink do more, which results in better care of it, despite the exhaustion and loss of control on personal needs. It should be noted that $100 \%$ of caregivers do not want to delegate the care of your family to others. (Items 14, 16, 17, 18, 20).

Significantly, the results of the 15 items concerning economic costs, which corroborates that this aspect is considered a major stressor in caregivers-relatives of patients with ESRD, as 8 caretakers equivalent to $88.2 \%$ of the population consider they do not have enough income to cover the costs of their family, and $11 \%$ (1 cuidadora) perceives it as sometimes.
In general, (4) caregivers representing $44 \%$, experience a degree of extreme loading, (3) caregivers, $33 \%$ and $22 \%$ much (2) moderate. Obviously $100 \%$ of the population feels overloaded which points the presence of psychological stress in caregivers (items 22). The degree of charge is related to the stage of the patient, income, age, presenting pathologies, kinship, and the perception they have about the disease, its care and fears about the uncertain future of the family.

\section{Session \#6}

\section{Symptomatic Stress Scale:}

With the application of this scale it was obtained that $100 \%$ of caregivers-relatives are stressed behaving symptoms as shown in Table \# 4 (See Annex \# 14). Symptoms prevail over each other, being the main ones: sleep disorder, lack of energy or depression, nervousness or anxiety, fatigue and weakness, headaches, palpitations or irregular heartbeat, excessive sweating, heartburn, irritability and loss of appetite. Confirmed what obtained in other techniques such as in-depth interview, the trait anxiety inventory as inventory status and Beck, demonstrating the psychological and physiological expression of stress in these caregivers.

\section{(See Exhibit \#15)}

In the analysis of this technique it is evident as reported by Reyes (2007) Arraras and Garrido (2008) in stating that primary caregivers exposed to anxiety and constant stress are more likely to exhibit disturbed sleep, loss of appetite, sedentary lifestyle among other manifestations that affect their immune function and has an impact on your health.

It is valid to note that for the application of this scale were directed at caregivers-family members should clarify whether these symptoms associated family situation or if other causes associated to age characteristics, such as menopause. ${ }^{8}$ In this case expression associated symptoms experiential states with the patient revealed a direct relationship between them and their performance as caretaker. ${ }^{9-13}$

Statistical analysis of this technique project through fashion as a measure of central tendency that features seven modes: sleep disorder, lack of energy or depression, nervousness or anxiety, fatigue and weakness, headaches, palpitations or irregular heartbeat and excessive sweating.

\section{Discussion and conclusions}

After done work sessions and integrate the results was obtained: 9 patients with chronic renal failure Terminal, ${ }^{14}$ the municipality Guise, are cared for by their closest relatives (daughters, mother, sisters, wives), highlighting once again the role of women in this work as well as the multiplicity of roles that characterize them as linked to the care of her sick have over them other household responsibilities. Have ages between 41 and 58 years of age, a period that puts them at the stage of middle adulthood, ${ }^{15}$ where you can appreciate the social situation of development in their actions, their own crisis of this stage as the "empty nest", hardship or loss , marital status (divorce) however, associated mood fluctuations, Although the research was conducted through a descriptive study, it was observed that the impact and stage of disease, the perception of care they offer, the diversity of family and personal situations that vivencian, the actual risk of infection generated by the disease, among other serious complications, influence the presence of psychological stress, and this in turn can promote the progress and development of chronic non-communicable diseases, which together 
with the age with this disease may present (IRC).

When analyzing the emerging situation (demand) they face caretakers, knowledge and perception (capacity) thereof, as well as the different styles of confrontation, highlighting behaviours of flight and evasion as direct styles, and some are seen styles indirect as: autorelajacion and psychotropic drugs, is also evident behavioural characteristics of different behaviour patterns, showing the relationship between these and stress. Essentially it could be perceived in the typical behaviours of caregiver's type A behaviour pattern, although we note that was present behaviour pattern type C.

Obtained general sense that $100 \%$ of caregivers ${ }^{16}$ reflect anxiety in the middle levels trait and state ( 3 caretakers) high levels trait and state (6 caregivers) symptoms of depression, expressed mainly in discontent, frequent crying, suicidal impulse , fatigue, caused by insufficient sleep; inapetencia that generated weight loss are symptoms that always accompany psychological stress. ${ }^{17-19}$ Uncertainty about death or future prospects of the family, lack of "respites" feeling that no truce conviction that being next to their family is their place and duty, "no one else can care as she does". At the same time significantly prevail the following manifestations of stress: anxiety, depression, sleep disorder, fatigue, headaches, sweating, and palpitations.

\section{After the theoretical analysis and practical observation made it is concluded that}

1. Women still figure responsible for the care of patients with ESRD, whose ages are between 41 and 58 years old, predominating as degree of kinship wives.

2. Caregivers-family corresponds to middle adulthood stage of development characterized by a set of crisis and discomfort associated with this, favouring the presence of psychological stress. $^{20}$

3. Psychological stress affects caregivers-relatives of patients with ESRD and its main manifestations are: anxiety, depression, sleeps disturbances, fatigue, headaches, loss of appetite, heartburn, palpitations and sweating, demonstrating the highest levels which take longer to play its role.

4. Patterns of behaviour, are predisposing factors to stress in these caregivers-family, essentially the Type A behaviour pattern

5. Caring for patients with ESRD, it influences the course and development of chronic non-communicable diseases such as Hypertension and Diabetes Mellitus affecting the quality of life of the caregiver and therefore the quality of care they offer.

\section{Recommendations}

The Department of Psychology ${ }^{21}$ of the municipality of Guise:

a. Design psychological intervention strategies aimed at caregivers-relatives of patients with ESRD in order to reduce the high levels of stress they are constantly subjected.

b. Perform this type of study other caregivers of dependent patients who may be victims of negative manifestations of psychological stress.

\section{Acknowledgements}

None.

\section{Conflict of interest}

The author declares there is no conflict of interest.

\section{References}

1. DG WinSock. Chronic renal failure. In: Bennett JC, Plum F. Treaty of Internal Medicine Cecil. Vol. I. Havana: Editorial of Medical Sciences. 1998;581-717.

2. Tobo N, Martinez G, Mosquera M. Compliance with medical regimen and their relationship are biological and social features of the individual with chronic renal failure terminal haemodialysis. Colombia Médica. 1995;26:141-145

3. Jacqueline Hernandez Almeida. Psychological Patient Care in Chronic Renal Failure Terminal. Digital support materials. 2009.

4. Public Health Statistical Yearbook 2017. Guise. Granma.

5. Martinez Otero Aidé. Women, stress and care of a family dependent Cuban magazine Sexology and Society. 2004;23(2).

6. Lazarus RS, Salkaam S. Stress and cognitive processes. Barcelona: Roca editions. 1986; p. 89.

7. Zaldivar P. Knowledge and mastery of stress. Havana. Editoria Technical scientific. p. 52

8. Anarte T. Menopause and stress. Digital support materials. p. 1.

9. Gomez M. Evolution of care for dependents. Digital support materials p. 5.

10. Frankly C. Who cares for the caregiver? 2002.

11. Losada A, Marques M, Peñacoba A. Reflections on care for caregivers of people with dementia and proposed an interdisciplinary intervention. Psychology behavioral. 2007;15(1):57-76.

12. Lee H, Camron M. Respite care for people with dementia and their caregivers. Cochrane Database Syst Rev. 2004;(2):CD004396.

13. Lavilla J. Who cares for the caregiver? 2004

14. DG WinSock. Chronic renal failure. In: Bennett JC, Plum F. Treaty of Medicine.

15. Febles M. A new stage of development: adulthood. Materials digital support.

16. Roca A, White K. Burden on caregivers madmen. 2007.

17. Stress and Cognitive Processes. (Sf) Chapter- 1. "The concept of stress on biology". Digital support materials. p.10.

18. Gonzalez M. Stress, a comprehensive approach. Havana: Editorial scientific-technical. 2000; p.68.

19. Slipack O. Stress and personality profiles. 2006 .

20. Carrazana Y. Psychological. Estrés workers in the area. 2008.

21. Business of Banco Popular de Ahorro de Guise. Thesis for the Degree in Psychology, University of Granma, SUM Guise. p.6-36. 